\title{
Inflammatorischer Pseudotumor der Lunge als seltene Differenzialdiagnose einer pulmonalen Raumforderung - ein Fallbericht mit Literaturübersicht
}

\author{
Inflammatory Pseudotumour of the Lung as a Rare Differential Diagnosis of a Pulmonary Mass - A Case Report \\ and Overview of the Literature
}

Autoren

Institut
M. C. Kleinschmidt, C. W. Scherbaum, T. Müller

Klinik für Pneumologie, Kliniken des Main-Taunus-Kreises, Hofheim am Taunus (Klinikleiter: T. Müller) eingereicht 18.8.2009

akzeptiert nach Revision

12. 10.2009

\section{Bibliografie}

DOI http://dx.doi.org/

10.1055/s-0029-1215289

Online-Publikation: 18.11. 2009

Pneumologie 2010; 64:

246-248 @ Georg Thieme

Verlag KG Stuttgart · New York

ISSN 0934-8387

Korrespondenzadresse

Dr. med. Malte Christian Kleinschmidt

Klinik für Pneumologie

Lindenstraße 10

65719 Hofheim/Taunus

mckleinschmidt@kliniken-mtk.de

\section{Zusammenfassung \\ $\nabla$}

Der Fall einer 37-jährigen Patientin mit einem inflammatorischen Pseudotumor der Lunge wird geschildert. Inflammatorische Pseudotumore der Lunge sind eine seltene, benigne Erkrankung, die vornehmlich jüngere Patienten betrifft. Aufgrund der Seltenheit sowie der häufig unspezifischen klinischen und radiologischen Befunde bereiten sie regelmäßig diagnostische und therapeutische Schwierigkeiten. Der Fallvorstellung folgt eine kurze Literaturübersicht.

\section{Einleitung}

$\nabla$

Der Fall einer 37-jährigen Patientin mit einem inflammatorischen Pseudotumor der Lunge wird geschildert. Inflammatorische Pseudotumore der Lunge sind eine seltene Erkrankung und bereiten dem klinisch tätigen Arzt regelmäßig diagnostische und therapeutische Schwierigkeiten. Es handelt sich dabei um einen vornehmlich jüngere Patienten betreffenden benignen Prozess unklarer Genese, der sich in der Regel als solide, scharf begrenzte Raumforderung manifestiert und charakterisiert ist durch eine unkontrollierte Proliferation inflammatorischer Immunzellen [1,2]. Obwohl, wie im von uns geschilderten Fall, etwa einem Drittel der Fälle ein Infekt der unteren Atemwege vorausgeht, ließ sich bisher kein infektiöses Agens als Auslöser identifizieren [2]. Insbesondere der häufig unspezifische histologische Befund erschwert die Diagnose und Abgrenzung zu anderen Krankheitsentitäten [3].

\section{Fallbericht}

$\nabla$

Eine 37-jährige Frau asiatischer Herkunft stellte sich mit Husten, Fieber und putridem Auswurf in der allgemein-internistischen Abteilung unserer Klinik vor. Sie berichtet von einer klinisch diag-

\section{Abstract \\ $\nabla$}

The case of a 37-year-old woman with an inflammatory pseudotumour of the lung is reported. Inflammatory pseudotumour of the lung is a rare disease predominantly occurring in younger patients. Because of its rarity as well as its usually unspecific clinical and radiological presentation, this disease constantly causes diagnostic and therapeutic difficulties. The case report is followed by a short overview of the literature.

nostizierten Pneumonie vor einem halben Jahr sowie einer durchgemachten, radiologisch nachgewiesenen Pneumonie vor 3,5 Jahren. Nikotinkonsum wird verneint. In der Röntgen-ThoraxAufnahme zeigte sich eine Infiltration des linken Lungenoberlappens ( $\bullet$ Abb. 1). Unter der Annahme einer ambulant-erworbenen Pneumonie erfolgte eine kalkulierte antibiotische Behandlung mit zunächst Cefuroxim i.v. und dann, bei Beschwerdepersistenz, kombiniert mit Cefuroxim und Clarithromycin i.v. Hierunter bildeten sich Infektwerte, Beschwerden und der radiologische Befund nicht wesentlich zurück, woraufhin uns die Patientin zur weiteren differenzialdiagnostischen Einordnung vorgestellt wurde.

\section{Klinischer Untersuchungsbefund}

Eine 37-jährige Frau asiatischer Herkunft in mäßig reduziertem Allgemeinzustand. Es fand sich ein vesikuläres Atemgeräusch. Die übrige körperliche Untersuchung erbrachte keine auffälligen Befunde.

\section{Apparative Untersuchungen}

In der durchgeführten Computertomografie stellte sich eine $6 \mathrm{~cm}$ messende, unregelmäßig begrenzte, lappenübergreifende solide Raumforderung der linken Lunge mit breitem Kontakt zur Thoraxwand dar, zentral relativ hypodens, im 


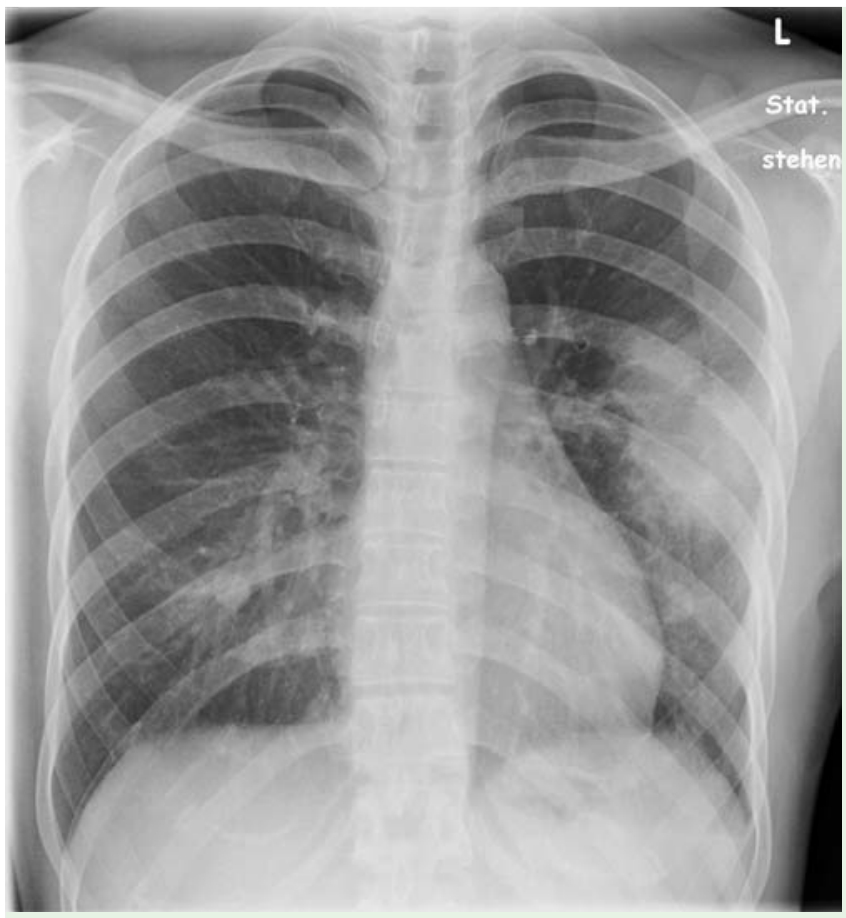

Abb. 1 37-jährige Patientin mit einem inflammatorischen Pseudotumor der Lunge, die über Husten, Auswurf und Fieber klagt. In der Röntgen-Thorax-Aufnahme (р. a.) findet sich eine umschriebene periphere Raumforderung im linken Ober- und Unterfeld.

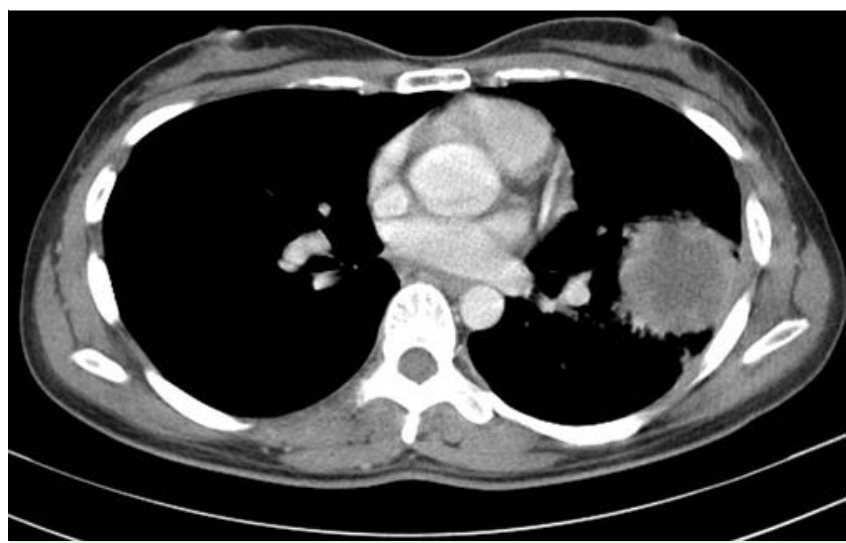

Abb. 2 Im CT-Thorax kommt eine $6 \mathrm{~cm}$ messende, unregelmäßig begrenzte, lappenübergreifende solide Raumforderung der linken Lunge mit breitem Kontakt zur Thoraxwand zur Darstellung.

Randbereich mit guter Kontrastmittelaufnahme ( $\mathbf{A b b . 2}$ ). Die hiliären und mediastinalen Lymphknoten waren nicht vergrößert, das übrige Lungenparenchym nicht pathologisch verändert. Die anschließende Bronchoskopie erbrachte einen Normalbefund. Es erfolgte eine zweifache transthorakale Biopsie der Raumforderung mit u.g. histologischen Ergebnis.

Klinisch-chemische, mikrobiologische und pathologische Befunde

Bei Übernahme fand sich ein erhöhtes CRP von $6,77 \mathrm{mg} / \mathrm{dl}$ (Normbereich $<0,5 \mathrm{mg} / \mathrm{dl}$ ), alle übrigen Standardlaborparameter lagen im Normbereich. Ein serologischer Nachweis M. tuberculosis-spezifischer T-Zellen (T-SPOT.TB, Oxford Immunotec Ltd.) gelang nicht, die laborchemischen Sarkoidose-Marker Calcium,

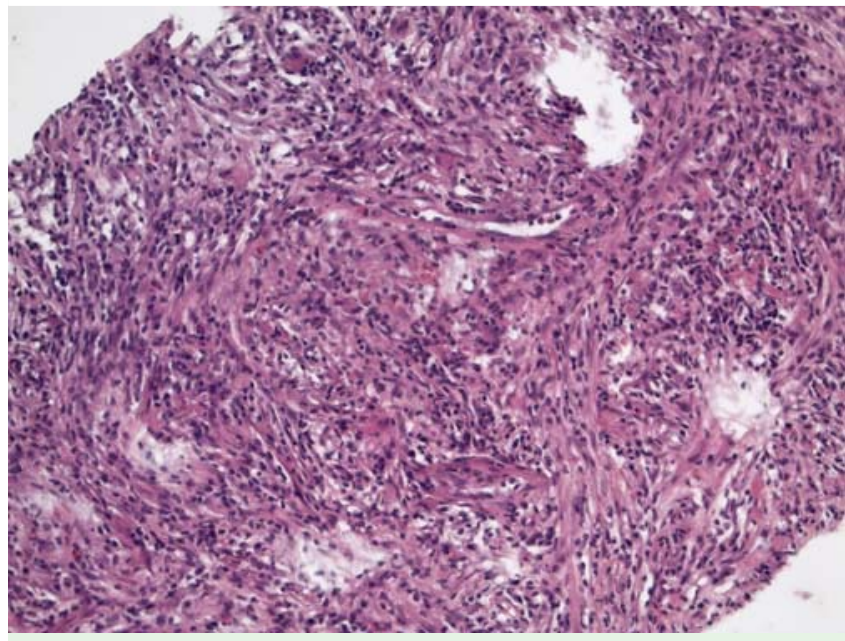

Abb. 3 Histologischer Aspekt der zweiten Stanzbiopsie (Vergrößerung $1: 100)$ : Schlanke, bündelartige fibroblastäre Proliferate mit einem lockeren Rundzellinfiltrat mit reichlich Plasmazellaggregaten.

ACE, löslicher IL-2-Rezeptor und Neopterin waren allesamt unauffällig. Ebenso fanden sich normwertige Serumkonzentrationen für die Tumormaker CEA, Cyfra 21-1 und NSE.

Die broncho-alveoläre Lavage erbrachte einen zytologischen Normalbefund mit einer CD4/8-Ratio von 1,2. Es fanden sich neben der physiologischen Flora mäßig Hämophilus parainfluenza sowohl im Sputum als auch im bronchoskopisch gewonnenen Trachealsekret ohne Nachweis säurefester Stäbchen (Mikroskopie und Kultur) bzw. M. tuberculosis-DNA.

In der histologischen Untersuchung des ersten transthorakal gewonnenen Stanzzylinders fanden sich nichtverkäsende epitheloidzellige Granulome. Eine zweite Stanzbiopsie zeigte schlanke, bündelartige fibroblastäre Proliferate mit einem lockeren Rundzellinfiltrat mit reichlich Plasmazellaggregaten ( $\mathbf{A b b}$. 3). Dieser zweite Befund wurde konsilarisch von einem universitären pathologischen Institut als inflammatorischer Pseudotumor vom fibroblastären Typ bestätigt.

\section{Therapie und Verlauf}

$\nabla$

Fußend auf dem initialen histologischen Nachweis epitheloidzelliger Granulom entschieden wir uns, mit Verdacht auf das Vorliegen einer atypischen Mykobakteriose für eine probatorische tuberkulostatische Vierfach-Therapie mit Isoniazid, Rifampicin, Pyrazinamid und Ciprofloxacin. Hierunter kam es nach vier Wochen zu einem signifikanten radiologischen Größenregress um ca. $30 \%$. Die klinischen Beschwerden waren, bis auf eine noch beklagte belastungsabhängige Atemnot, ebenfalls deutlich rückläufig.

Aufgrund des guten Ansprechens beschlossen wir, die Therapie weitere vier Wochen fortzuführen. Innerhalb dieser Zeit kam es zu keiner weiteren Größenreduktion, sodass wir, insbesondere nach Eintreffen der histologischen Endergebnisse und negativen Mykobakterienkulturen, die Differenzialdiagnose einer atypischen Mykobakteriose verwarfen und eine orale immunmodulatorische Therapie mit $50 \mathrm{mg}$ Prednisolon täglich begannen.

Hierunter kam es zu einer weiteren Größenreduktion des Tumors. Nach weiteren vier Wochen reduzierten wir das Kortison auf $25 \mathrm{mg}$ täglich und schlichen es innerhalb der folgenden vier Wochen gänzlich aus. 
Fünf Monate nach Erstvorstellung fand sich noch ein ca. $2 \mathrm{~cm}$ messender Restbefund, nach Absetzen des Kortisons kam es nicht zu einer Befundverschlechterung. Es erfolgte eine chirurgische Weiterbehandlung mit offen chirurgischer Keilresektion des Pseudotumors infolge. Histologisch bestätigte sich im Resektat die Diagnose eines inflammatorischen Pseudotumors. Der postoperative Verlauf gestaltete sich komplikationslos.

\section{Diskussion \\ $\nabla$}

Inflammatorische Pseudotumore können in nahezu allen Organen auftreten [4]. Angaben zur pulmonalen Inzidenz reichen von 0,04 bis $0,7 \%$ der pulmonalen Tumore, wobei die Erkankung das Lungenparenchym, das Bronchialsystem und die Pleura betreffen kann $[3,1]$.

Obwohl die Erkrankung grundsätzlich in allen Altersgruppen auftritt, sind vornehmlich jüngere Patienten betroffen. Es ist der häufigste benigne Tumor der Lunge im Kleinkindesalter, etwa die Hälfte der bisher beschriebenen Fälle trat vor dem 40. Lebensjahr auf, $15 \%$ im Alter von 1 - 10 Jahren [5]. Ob es eine geschlechtsspezifische Präferenz gibt, wird in der Literatur kontrovers diskutiert; in einer Studie mit 28 Fällen waren männliche Patienten mit 81,5\% deutlich bevorzugt betroffen [1].

Die beklagten Beschwerden sind in der Regel Husten, Fieber und Auswurf. Dyspnoe, Hämoptysen sowie thorakale Schmerzen können ebenfalls, wenn auch seltener, auftreten $[1,6]$.

Die radiologischen Erscheinungsformen pulmonaler inflammatorischer Pseudotumore wurden u.a. von Agrons et al. ausführlich beschrieben [7]. Es handelt sich zumeist um solitäre Raumforderungen oder Rundherde variabler Größe, die im konventionellen Röntgenbild typischerweise umschrieben und scharf begrenzt sind. Multiple Läsionen wurden in 5\% der Fälle gefunden, selten kommt es zur sekundären Infiltration von hiliären, mediastinalen, pleuralen und bronchialen Strukturen [7]. Kalzifikation oder Kavernenbildung wurde ebenfalls beschrieben, ist aber insgesamt sehr selten [7]. Grundsätzlich gibt es keine spezifischen radiologischen Veränderungen, weshalb die Abgrenzung insbesondere zu malignen Prozessen wesentlich ist $[6,7]$.

Die Genese inflammatorischer Pseudotumore ist nicht endgültig geklärt. Es wird angenommen, dass es sich um das Resultat einer reaktiven, überschießenden Proliferation inflammatorischer Immunzellen handelt [6]. Der Erkrankung gehen in etwa 30\% der Fälle Infekte der unteren Atemwege voraus, weshalb eine infektiöse Genese mehrfach postuliert wurde. Ein kausaler infektiöser Erreger ließ sich bisher allerdings nicht identifizieren $[3,6]$. In einer größeren Untersuchung von 32 Fällen sahen Matsubara et al. eine ätiologische Beziehung zur organisierenden Pneumonie und beschrieben einige histologische Gemeinsamkeiten [2].

Histologisch finden sich inflammatorische und mesenchymale Zellen (Plasmazellen, Histiozyten, Lymphozyten, Pneumozyten und spindelzellige Anteile), in variabler Zusammensetzung [8]. Entsprechend dem dominierenden Zelltypus werden grundsätzlich zwei Formen inflammatorischer Pseudotumore unterschie- den: der plasmazellreiche und der histiozytäre (fibroblastäre) Typ. Die Diagnose ist, wie im vorliegenden Fall, mitunter erschwert durch den häufig unspezifischen histologischen Befund, weshalb die endgültige pathologische Zuordnung oftmals erst nach Tumorextirpation gelingt [3,7].

Die Therapie der Wahl pulmonaler inflammatorischer Pseudotumore besteht in der operativen Entfernung. Wurden alle Tumoranteile entfernt, ist die Langzeitprognose sehr gut [8]. Weitere Optionen, z.B. bei funktioneller Inoperabilität oder insbesondere auch bei unvollständiger Tumorresektion, sind die medikamentöse Behandlung mit Kortikoiden oder auch der Tumorbestrahlung $[6,9,10]$. Ein Ansprechen der Erkrankung auf eine antibiotische Therapie, wie auch im vorliegenden Fall beobachtet, wurde bisher nur in Einzelfällen beschrieben [10]. Rezidive, obwohl äußerst selten, können auftreten [6].

Zusammenfassend handelt es sich beim inflammtorischen Pseudotumor der Lunge um eine seltene, grundsätzlich gutartige Erkrankung unklarer Genese, die bei der differenzialdiagnostischen Einordnung pulmonaler Raumforderungen, insbesondere bei jüngeren Patienten, berücksichtigt werden sollte. Die Abgrenzung zu anderen - auch malignen Prozessen - ist aufgrund der häufig unspezifischen klinischen, radiologischen und histologischen Befunde schwierig, weshalb die definitive Diagnose oft erst nach operativer Tumorentfernung gelingt.

\section{Interessenkonflikte}

$\nabla$

Die Autoren geben an, dass kein Interessenkonflikt besteht.

\section{Literatur}

$1 \mathrm{Kim} J \mathrm{H}$, Cho JH, Park MS et al. Pulmonary inflammatory pseudotumor a report of 28 cases. Korean J Intern Med 2002; 17: 252 - 258

2 Matsubana O, Tan-Liu NS, Kenney RM et al. Inflammatory pseudotumor of the lung: progression from organizing pneumonia to fibrous histiocytoma or plasma cell granuloma in 32 cases. Hum Pathol 1988; 19: $807-814$

3 Singh RS, Dhaliwal RS, Puri D et al. Inflammatory pseudotumour of the lung: report of a case and review of literature. Indian J Chest Dis Allied Sci 2001; $43: 31-234$

4 Narla LD, Newman B, Spottswood SS et al. Inflammatory pseudotumor. Radiographics 2003; 23: 719-729

5 Hartman GE, Shochat SJ. Primary pulmonary neoplasms of childhood: a review. Ann Thorac Surg 1983; 36: 108 -119

6 Patel SB, Shah DM, Goswami KG et al. Inflammatory pseudotumor of lung, a case report and review of literature. Ind J Radiol Imag 2006; 16: $117-118$

7 Agrons GA, Rosado-de-Christenson ML, Kirejczyk WM et al. Pulmonary inflammatory pseudotumor: radiologic features. Radiology 1998; 206: $511-518$

8 Dahabreh J, Zisis C, Arnogiannaki $N$ et al. Inflammatory pseudotumor: a controversial entity. J Cardiothorac Surg 1999; 16: 670-673

9 Fabre D, Fadel E, Singhal $S$ et al. Complete resection of pulmonary inflammatory pseudotumors has excellent long-term prognosis. J Thorac Cardiovasc Surg 2009; 137: 435-440

10 Piroth L, Menecier P, Charvillat L et al. Diagnostic and therapeutic approach to plasma cell granuloma of the lung. Apropos of a case of favourable course under antibiotics. Rev Med Interne 1996; 17: 670 674 